\title{
Necessary Conditions for the Application of Moving Average Process of Order Three
}

\author{
O. E. Okereke1, I. S. Iwueze'2, J. Ohakwe ${ }^{3}$ \\ ${ }^{1}$ Department of Statistics, Michael Okpara University of Agriculture, Umudike, Nigeria \\ ${ }^{2}$ Department of Statistics, Federal University of Technology, Owerri, Nigeria \\ ${ }^{3}$ Department of Mathematical, Computer and Physical Sciences, Federal University, Otueke, Nigeria \\ Email: emmastat5000@yahoo.co.uk
}

Received 26 November 2014; accepted 12 December 2014; published 19 January 2015

Copyright (C) 2015 by authors and Scientific Research Publishing Inc.

This work is licensed under the Creative Commons Attribution International License (CC BY).

http://creativecommons.org/licenses/by/4.0/

(c) (i) Open Access

\section{Abstract}

Invertibility is one of the desirable properties of moving average processes. This study derives consequences of the invertibility condition on the parameters of a moving average process of order three. The study also establishes the intervals for the first three autocorrelation coefficients of the moving average process of order three for the purpose of distinguishing between the process and any other process (linear or nonlinear) with similar autocorrelation structure. For an invertible moving average process of order three, the intervals obtained are $\frac{-1-\sqrt{5}}{4}<\rho_{1}<\frac{1-\sqrt{5}}{4}$, $-0.5<\rho_{2}<0.5$ and $-0.5<\rho_{1}<0.5$.

\section{Keywords}

Moving Average Process of Order Three, Characteristic Equation, Invertibility Condition, Autocorrelation Coefficient, Second Derivative Test

\section{Introduction}

Moving average processes (models) constitute a special class of linear time series models. A moving average process of order $q$ (MA $(q)$ process) is of the form:

$$
X_{t}=\theta_{1} e_{t-1}+\theta_{2} e_{t-2}+\cdots+\theta_{q} e_{t-q}+e_{q}
$$

where $\theta_{1}, \theta_{2}, \cdots, \theta_{q}$ are real constants and $e_{t}, t \in Z$ is a sequence of independent and identically distributed random variables with zero mean and constant variance. These processes have been widely used to model 
time series data from many fields [1]-[3]. The model in (1.1) is always stationary. Hence, a required condition for the use of the moving average process is that it is invertible. Let $B^{m} e_{t}=e_{t-m}$, then the model in (1.1) is invertible if the roots of the characteristic equation

$$
1+\theta_{1} B+\theta_{2} B^{2}+\cdots+\theta_{q} B^{q}=0
$$

lie outside the unit circle. The invertibility conditions of the first order and second order moving average models have been derived [4] [5].

Ref. [6] used a moving average process of order three (MA (3) process) in his simulation study. Though, higher order moving average processes have been used to model time series data, not much has been said about the properties of their autocorrelation functions. This study focuses on the invertibility condition of an MA (3) process. Consideration is also given to the properties of its autocorrelation coefficients of an invertible moving average process of order three.

\section{Consequence of Invertibility Condition on the Parameters of an MA (3) Process}

For $q=3$, the following moving average process of order 3 is obtained from (1.1):

$$
X_{t}=\theta_{1} e_{t-1}+\theta_{2} e_{t-2}+\theta_{3} e_{t-3}+e_{t}
$$

The characteristic equation corresponding to (2.1) is given by

$$
1+\theta_{1} B+\theta_{2} B^{2}+\theta_{3} B^{3}=0
$$

Dividing (2.2) by $\theta_{3}$ yields

$$
B^{3}+\frac{\theta_{2}}{\theta_{3}} B^{2}+\frac{\theta_{1}}{\theta_{3}} B+\frac{1}{\theta_{3}}=0
$$

It is important to know that (2.2) is a cubic equation. Detailed information on how to solve cubic equations can be found in [7] [8] among others. It has been a common tradition to consider the nature of the roots of a characteristic equation while determining the invertibility condition of a time series model [9]. As a cubic equation, (2.2) may have three distinct real roots, one real root and two complex roots, two real equal roots or three real equal roots. The nature of the roots of (2.2) is determined with the help of the discriminant [8]

$$
D=D_{1}^{2}-D_{2}^{3}
$$

where

$$
D_{1}=\frac{2\left(\frac{\theta_{2}}{\theta_{3}}\right)^{3}-9\left(\frac{\theta_{2}}{\theta_{3}}\right)\left(\frac{\theta_{1}}{\theta_{3}}\right)+27\left(\frac{1}{\theta_{3}}\right)}{54}
$$

and

$$
D_{2}=\frac{\left(\frac{\theta_{2}}{\theta_{3}}\right)^{2}-3\left(\frac{\theta_{1}}{\theta_{3}}\right)}{9}
$$

If $D<0,(2.2)$ has the following distinct roots [7]

$$
\begin{gathered}
x_{1}=-2 \sqrt{D_{2}} \cos \left(\frac{\theta}{3}\right)-\frac{\theta_{2}}{3}, \\
x_{2}=-2 \sqrt{D_{2}} \cos \left(\frac{\theta+2 \pi}{3}\right)-\frac{\theta_{2}}{3},
\end{gathered}
$$


and

$$
x_{3}=-2 \sqrt{D_{2}} \cos \left(\frac{\theta-2 \pi}{3}\right)-\frac{\theta_{2}}{3} .
$$

where $\theta$ is measured in radians and $\theta=\cos ^{-1}\left(\frac{D_{1}}{\sqrt{D_{2}^{3}}}\right)$.

When $D>0$, (2.2) has only real root given by [1] as

$$
x_{1}=\sqrt[3]{-D_{1}+\sqrt{D}}+\sqrt[3]{-D_{1}-\sqrt{D}}-\frac{\theta_{2}}{3}
$$

The other roots are [8]

$$
x_{2}, x_{3}=\frac{-\left(a x_{1}+b\right) \pm \sqrt{\left(a x_{1}+b\right)^{2}-4 a\left(a x_{1}^{2}+b x_{1}+c\right)}}{2 a}
$$

If $D_{1} \neq 0, D_{2} \neq 0$ and $D_{1}^{2}=D_{2}^{3}$, then $D=0$ and (2.2) has two equal roots. The roots of (2.2) in this case, are the same as (2.7), (2.8) and (2.9). For $D=0$ and $D_{1}=D_{2}=0,(2.2)$ has three real equal roots. Each of these roots is given by [8] as

$$
x=\frac{-\theta_{2}}{3 \theta_{3}}
$$

For (2.1) to be invertible, the roots of (2.2) are all expected to lie outside the unit circle and $\left|\theta_{3}\right|<1$. In the following theorem, the invertibility conditions of an MA (3) process are given subject to the condition that the corresponding characteristic equation has three real equal roots.

Theorem 1. If the characteristic equation $1+\theta_{1} B+\theta_{2} B^{2}+\theta_{3} B^{3}=0$ has three real equal roots, then the moving average process of order three $X_{t}=\theta_{1} e_{t-1}+\theta_{2} e_{t-2}+\theta_{3} e_{t-3}+e_{t}$ is invertible if

$$
\theta_{2}-3 \theta_{3}>0, \theta_{2}+3 \theta_{3}<0 \text { and }\left|\theta_{3}\right|<1 \text {. }
$$

Proof

For invertibility, we expect each of the three real equal roots to lie outside the unit circle. Thus,

$$
\left|\frac{-\theta_{2}}{3 \theta_{3}}\right|>1 \Rightarrow \frac{-\theta_{2}}{3 \theta_{3}}<-1 \text { or } \frac{-\theta_{2}}{3 \theta_{3}}>1
$$

Solving the inequality $\frac{-\theta_{2}}{3 \theta_{3}}<-1$, we obtain

$$
\theta_{2}-3 \theta_{3}>0
$$

For $\frac{-\theta_{2}}{3 \theta_{3}}>1$, we have

$$
\theta_{2}+3 \theta_{3}<0
$$

Since each of the roots lie outside the unit circle, the absolute value of their product must therefore be greater than one. Hence,

$$
\left|\theta_{3}\right|<1
$$

This completes the proof.

The invertibility region of a moving average of order three with equal roots of the characteristic Equation (2.2) is enclosed by triangle OAB in Figure 1. 


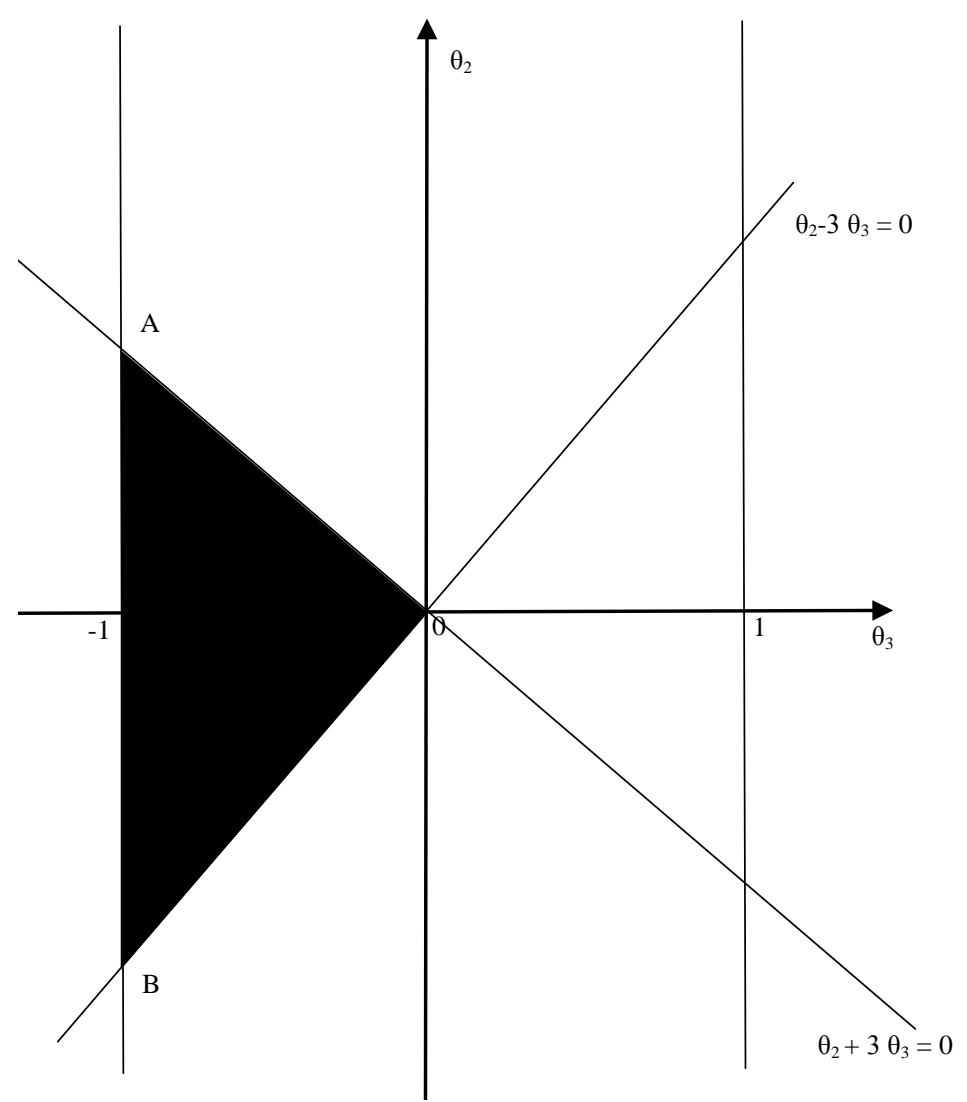

Figure 1. Invertibility region of an MA (3) process when the characteristic equation has three real equal roots.

\section{Identification of Moving Average Process}

Model identification is a crucial aspect of time series analysis. A common practice is to examine the structures of the autocorrelation function (ACF) and partial autocorrelation function (PACF) of a given time series. In this regard, a time series is said to follow a moving average process of order $q$ if its associated autocorrelation function cut off after lag $q$ and the corresponding partial autocorrelation function decays exponentially [10]. Authors using this method, believe that each process has unique ACF representation. However, the existence of similar autocorrelation structures between moving average process and pure diagonal bilinear time series process of the same order makes it difficult to identify a moving average process based on the pattern of its ACF. Furthermore, a careful look at the autocorrelation function of the square of a time series can help one determine if the series follows a moving average process. If the series can be generated by a moving average process, then its square follows a moving average process of the same order [11] [12]. The conditions under which we use the autocorrelation function to distinguish among processes behaving like moving average processes of order one and two have been determined by [13] [14] respectively. These conditions are all defined in terms of the extreme values of autocorrelation coefficients of the processes.

\section{Intervals for Autocorrelation Coefficients of a Moving Average Process of Order Three}

As stated in Section 3, knowledge of the extreme values of the autocorrelation coefficient of a moving average process of a particular order can enable us ensure proper identification of the process. It has been observed that for a moving average process of order one, $-0.5 \leq \rho_{1} \leq 0.5$ [15] while for a moving average process of order two $-\frac{\sqrt{2}}{2} \leq \rho_{1} \leq \frac{\sqrt{2}}{2}$ and $-0.5 \leq \rho_{2} \leq 0.5$ [5]. In order to generalize about the range of values of $\rho_{q}$ for a 
moving average process of order $q$, it is worthwhile to determine the range values of $\rho_{3}$ for a moving average process of order three. The model in (2.1) has the following autocorrelation function [10]:

$$
\rho_{k}= \begin{cases}1, & k=0 \\ \frac{\theta_{1}+\theta_{1} \theta_{2}+\theta_{2} \theta_{3}}{1+\theta_{1}^{2}+\theta_{2}^{2}+\theta_{3}^{2}}, & k= \pm 1 \\ \frac{\theta_{2}+\theta_{1} \theta_{3}}{1+\theta_{1}^{2}+\theta_{2}^{2}+\theta_{3}^{2}}, & k= \pm 2 \\ \frac{\theta_{3}}{1+\theta_{1}^{2}+\theta_{2}^{2}+\theta_{3}^{2}}, & k= \pm 3 \\ 0, & k \neq \pm 1, \pm 2, \pm 3\end{cases}
$$

We can deduce from (4.1) that the autocorrelation function at lag one of the MA (3) process is

$$
\rho_{1}=\frac{\theta_{1}+\theta_{1} \theta_{2}+\theta_{2} \theta_{3}}{1+\theta_{1}^{2}+\theta_{2}^{2}+\theta_{3}^{2}}
$$

Using the Scientific Note Book, the minimum and maximum values of $\rho_{1}$ are found to be $\frac{-1-\sqrt{5}}{4}$ and $\frac{1-\sqrt{5}}{4}$ respectively. For the autocorrelation function at lag two, we have

$$
\rho_{2}=\frac{\theta_{2}+\theta_{1} \theta_{3}}{1+\theta_{1}^{2}+\theta_{2}^{2}+\theta_{3}^{2}}
$$

The extreme values of $\rho_{2}$ are equally obtained with the help of the Scientific Note Book. To this effect, $\rho_{2}$ has a minimum value of -0.5 and a maximum value of 0.5 .

From (4.1), we obtain

$$
\rho_{3}=\frac{\theta_{3}}{1+\theta_{1}^{2}+\theta_{2}^{2}+\theta_{3}^{2}}
$$

Based on the result obtained from the Scientific Notebook, $\rho_{3}$ has a minimum value of -0.5 and a maximum value of 0.5 . However, the intervals for $\rho_{3}$ can easily be obtained analytically and this result is generalized in Theorem 2 for $\rho_{q}$ of the MA $(q)$ process.

The partial derivatives of $\rho_{3}$ with respect to $\theta_{1}, \theta_{2}$ and $\theta_{3}$ are

$$
\begin{aligned}
& \frac{\partial \rho_{3}}{\partial \theta_{1}}=\frac{-2 \theta_{1} \theta_{3}}{\left(1+\theta_{1}^{2}+\theta_{2}^{2}+\theta_{3}^{2}\right)^{2}} \\
& \frac{\partial \rho_{3}}{\partial \theta_{2}}=\frac{-2 \theta_{2} \theta_{3}}{\left(1+\theta_{1}^{2}+\theta_{2}^{2}+\theta_{3}^{2}\right)^{2}} \\
& \frac{\partial \rho_{3}}{\partial \theta_{3}}=\frac{1+\theta_{1}^{2}+\theta_{2}^{2}-\theta_{3}^{2}}{\left(1+\theta_{1}^{2}+\theta_{2}^{2}+\theta_{3}^{2}\right)^{2}}
\end{aligned}
$$

The critical points of $\rho_{3}$ occurs when $\frac{\partial \rho_{3}}{\partial \theta_{i}}=, i=1,2,3$. Equating each of the partial derivatives in (4.5), (4.6) and (4.7) to zero, we obtain

$$
\theta_{1} \theta_{3}=0
$$




$$
\begin{gathered}
\theta_{2} \theta_{3}=0 \\
1+\theta_{1}^{2}+\theta_{2}^{2}-\theta_{3}^{2}=0
\end{gathered}
$$

From (4.10), we have

$$
\theta_{3}= \pm \sqrt{1+\theta_{1}^{2}+\theta_{2}^{2}}
$$

Using (4.8), we obtain

$$
\theta_{1}=0
$$

or

$$
\theta_{3}=0
$$

Substituting $\theta_{1}=0$ into (4.11) yields

$$
\theta_{3}= \pm \sqrt{1+\theta_{2}^{2}}
$$

For $\theta_{3}=-\sqrt{1+\theta_{2}^{2}}$, (4.9) becomes

$$
\begin{gathered}
\theta_{2}\left(\sqrt{1+\theta_{2}^{2}}\right)=0 \\
\theta_{2}^{2}\left(1+\theta_{2}^{2}\right)=0 \\
\theta_{2}=0 \text { or } \theta_{2}= \pm \sqrt{-1}
\end{gathered}
$$

If we also substitute $\theta_{3}=\sqrt{1+\theta_{2}^{2}}$ into (4.9), we obtain

$$
\theta_{2}=0 \text { or } \theta_{2}= \pm \sqrt{-1}
$$

When we substitute $\theta_{1}=0$ and $\theta_{2}=0$ into (4.11), we have $\theta_{3}= \pm 1$. It is also clear that if $\theta_{1}=0$ and $\theta_{2}=-\sqrt{-1}$, then $\theta_{3}=0$. Similar result is obtained when $\theta_{1}=0$ and $\theta_{2}=\sqrt{-1}$.

Hence, the critical points of $\rho_{3}$ are $(0,0,-1),(0,0,1),(0,-\sqrt{-1}, 0)$ and $(0, \sqrt{-1}, 0)$.

The minimum and maximum values of a function occur at it critical points. To determine which of the critical points is a local minimum, local maximum or a saddle point, we shall apply the second derivative test. The second derivative test for critical points of a function of three variables $\rho_{3}=f(x, y, z)$ focuses on the Hessian matrix:

$$
H=\left[\begin{array}{lll}
f_{x x} & f_{x y} & f_{x z} \\
f_{x y} & f_{y y} & f_{y z} \\
f_{x z} & f_{y z} & f_{z z}
\end{array}\right]
$$

where

$$
\begin{aligned}
& f_{x x}=\frac{\partial^{2} \rho_{3}}{\partial \theta_{1}^{2}}=\frac{-2 \theta_{3}\left(1+\theta_{1}^{2}+\theta_{2}^{2}+\theta_{3}^{2}\right)+8 \theta_{1} \theta_{3}^{2}}{\left(1+\theta_{1}^{2} \theta_{2}^{2} \theta_{3}^{2}\right)^{3}} \\
& f_{x y}=\frac{\partial^{2} \rho_{3}}{\partial \theta_{1} \partial \theta_{2}}=\frac{-8 \theta_{1} \theta_{2} \theta_{3}}{\left(1+\theta_{1}^{2} \theta_{2}^{2} \theta_{3}^{2}\right)^{3}} \\
& f_{x z}=\frac{\partial^{2} \rho_{3}}{\partial \theta_{1} \partial \theta_{3}}=\frac{-2 \theta_{1}\left(1+\theta_{1}^{2}+\theta_{2}^{2}+\theta_{3}^{2}\right)+8 \theta_{1} \theta_{3}^{2}}{\left(1+\theta_{1}^{2} \theta_{2}^{2} \theta_{3}^{2}\right)^{3}}
\end{aligned}
$$




$$
\begin{aligned}
f_{y y} & =\frac{-2 \theta_{3}\left(1+\theta_{1}^{2}+\theta_{2}^{2}+\theta_{3}^{2}\right)+8 \theta_{2}^{2} \theta_{3}}{\left(1+\theta_{1}^{2} \theta_{2}^{2} \theta_{3}^{2}\right)^{3}} \\
f_{y z} & =\frac{-2 \theta_{2}\left(1+\theta_{1}^{2}+\theta_{2}^{2}+\theta_{3}^{2}\right)+8 \theta_{2} \theta_{3}^{2}}{\left(1+\theta_{1}^{2} \theta_{2}^{2} \theta_{3}^{2}\right)^{3}} \\
f_{z z} & =\frac{-2 \theta_{3}\left(1+\theta_{1}^{2} \theta_{2}^{2} \theta_{3}^{2}\right)-4 \theta_{3}\left(1+\theta_{1}^{2}+\theta_{2}^{2}-\theta_{3}^{2}\right)}{\left(1+\theta_{1}^{2} \theta_{2}^{2} \theta_{3}^{2}\right)^{3}}
\end{aligned}
$$

Let $(a, b, c)$ be a critical point of $\rho_{3}=f(x, y, z)$. Then $(a, b, c)$ is called a local minimum point if at $(a, b, c), \Delta_{1}=f_{x x}>0, \Delta_{2}=\left|\begin{array}{ll}f_{x x} & f_{x y} \\ f_{x y} & f_{y y}\end{array}\right|>0$ and $\Delta_{3}=|H|>0$ [16]. If $f_{x x}<0, \Delta_{2}>0$ and $\Delta_{3}<0$ at $(a, b, c)$, then $(a, b, c)$ represents a local maximum.

A critical point that is neither a local minimum nor a local maximum is called a saddle point.

Though $\rho_{3}$ has four critical points, it is not defined at $(0,-\sqrt{-1}, 0)$ and $(0, \sqrt{-1}, 0)$. We then focus on the classification of the two remaining critical points.

At $(0,0,-1)$

$$
H=\left[\begin{array}{ccc}
\frac{1}{2} & 0 & 0 \\
0 & \frac{1}{2} & 0 \\
0 & 0 & \frac{1}{2}
\end{array}\right]
$$

Hence, $\Delta_{1}=\frac{1}{2}>0, \Delta_{2}=\left|\begin{array}{cc}\frac{1}{2} & 0 \\ 0 & \frac{1}{2}\end{array}\right|=\frac{1}{4}>0$ and $\Delta_{3}=\left|\begin{array}{ccc}\frac{1}{2} & 0 & 0 \\ 0 & \frac{1}{2} & 0 \\ 0 & 0 & \frac{1}{2}\end{array}\right|=\frac{1}{8}>0$.

Therefore, $(0,0,-1)$ is a local minimum. The value of $\rho_{3}$ at this point is -0.5 .

For the critical points $(0,0,1)$, we have

$$
H=\left[\begin{array}{ccc}
-\frac{1}{2} & 0 & 0 \\
0 & -\frac{1}{2} & 0 \\
0 & 0 & -\frac{1}{2}
\end{array}\right]
$$

Consequently,

$$
\begin{gathered}
\Delta_{1}=-\frac{1}{2}<0, \\
\Delta_{2}=\left|\begin{array}{cc}
-\frac{1}{2} & 0 \\
0 & -\frac{1}{2}
\end{array}\right|=\frac{1}{4}>0
\end{gathered}
$$


and

$$
\Delta_{3}=\left|\begin{array}{ccc}
-\frac{1}{2} & 0 & 0 \\
0 & -\frac{1}{2} & 0 \\
0 & 0 & -\frac{1}{2}
\end{array}\right|=-\frac{1}{8}<0 .
$$

We therefore conclude that $(0,0,1)$ is a local maximum. The maximum value of $\rho_{3}$ obtained at $(0,0,1)$ is 0.5 .

We can deduce from the result in this section and other previous works that for MA (1) process $\left|\rho_{1}\right| \leq 0.5$, while for MA (2) process and MA (3) process $\left|\rho_{2}\right| \leq 0.5$ and $\left|\rho_{3}\right| \leq 0.5$ respectively.

In what follows, we establish the bounds for $\rho_{q}$, where $q$ is order of the moving average process.

\section{Theorem 2.}

Let $X_{t}=\theta_{1} e_{t-1}+\theta_{2} e_{t-2}+\cdots+\theta_{q} e_{t-q}+e_{q}$ be an MA $(q)$ process. Then, $\left|\rho_{q}\right| \leq 0.5$.

Proof

It is easily seen that for the MA $(q)$ process,

$$
\rho_{q}=\frac{\theta_{q}}{1+\theta_{1}^{2}+\theta_{2}^{2}+\cdots+\theta_{q}^{2}}
$$

Partial derivatives of $\rho_{q}$ with respect to $\rho_{q}=\theta_{1}, \theta_{2}, \cdots, \theta_{q}$ are as follows

$$
\begin{gathered}
\frac{\partial \rho_{q}}{\partial \theta_{1}}=\frac{-2 \theta_{1} \theta_{q}}{\left(1+\theta_{1}^{2}+\theta_{2}^{2}+\cdots+\theta_{q}^{2}\right)^{2}}, \\
\frac{\partial \rho_{q}}{\partial \theta_{2}}=\frac{-2 \theta_{2} \theta_{q}}{\left(1+\theta_{1}^{2}+\theta_{2}^{2}+\cdots+\theta_{q}^{2}\right)^{2}}, \\
\vdots \\
\frac{\partial \rho_{q}}{\partial \theta_{q-1}}=\frac{-2 \theta_{q-1} \theta_{q}}{\left(1+\theta_{1}^{2}+\theta_{2}^{2}+\cdots+\theta_{q}^{2}\right)^{2}}, \\
\frac{\partial \rho_{q}}{\partial \theta_{q}}=\frac{1+\theta_{1}^{2}+\theta_{2}^{2}+\cdots+\theta_{q-1}^{2}-\theta_{q}^{2}}{\left(1+\theta_{1}^{2}+\theta_{2}^{2}+\cdots+\theta_{q}^{2}\right)^{2}} .
\end{gathered}
$$

Equating each of the partial derivatives to zero yields

$$
\begin{aligned}
& -2 \theta_{1} \theta_{q}=0, \\
& -2 \theta_{2} \theta_{q}=0, \\
& \quad \vdots \\
& -2 \theta_{q-1} \theta_{q}=0, \\
& 1+\theta_{1}^{2}+\theta_{2}^{2}+\cdots+\theta_{q-1}^{2}-\theta_{q}^{2}=0 .
\end{aligned}
$$

From (4.24), we obtain

$$
\theta_{q}= \pm \sqrt{1+\theta_{1}^{2}+\theta_{2}^{2}+\cdots-\theta_{q-1}^{2}}
$$

Since $\theta_{q} \neq 0$ for an MA $(q)$ process, it is obvious that the $q-1$ equations preceding (4.24) are only satisfied if $\theta_{1}=\theta_{2}=\cdots=\theta_{q-1}=0$. Substituting $\theta_{1}=\theta_{2}=\cdots=\theta_{q-1}=0$ into (4.25) leads to $\theta_{q}= \pm 1$. The two critical points of $\rho_{q}$ are then $(0,0,0, \cdots,-1)$ and $(0,0,0, \cdots, 1)$. 
At $(0,0,0, \cdots,-1), \rho_{q}=-0.5$ while at $(0,0,0, \cdots, 1), \rho_{q}=0.5$. It then follows that $\left|\rho_{\mathrm{q}}\right| \leq 0.5$.

Remark: For an invertible MA (3) process, $\left|\theta_{3}\right|<1$. Hence, $\frac{-1-\sqrt{5}}{4}<\rho_{1}<\frac{1-\sqrt{5}}{4},-0.5<\rho_{2}<0.5$ and $-0.5<\rho_{1}<0.5$.

\section{Conclusion}

We have established necessary conditions for the parameters of an invertible MA (3) process. When the characteristic equation has three real equal roots, the conditions are $\theta_{2}-3 \theta_{3}>0, \theta_{2}+3 \theta_{3}<0$ and $\left|\theta_{3}\right|<1$. Also the intervals for the autocorrelation coefficients of an invertible moving average process of order three are established. These are $\frac{-1-\sqrt{5}}{4}<\rho_{1}<\frac{1-\sqrt{5}}{4},-0.5<\rho_{2}<0.5$ and $-0.5<\rho_{1}<0.5$. It is also noteworthy that the condition on $\rho_{3}$ for an invertible MA (3) process is generalized for $\rho_{q}$ of the invertible MA $(q)$ process. That is for the invertible MA $(q)$ process, $\left|\rho_{\mathrm{q}}\right|<0.5$. These results can now be used to compare other linear and nonlinear processes that have similar autocorrelation structures with the MA (3) process.

\section{References}

[1] Moses, R.L. and Liu, D. (1991) Optimal Nonnegative Definite Approximation of Estimated Moving Average Covariance Sequences. IEEE Transactions on Signal Processing, 39, 2007-2015. http://dx.doi.org/10.1109/78.134433

[2] Qian, G. and Zhao, X. (2007) On Time Series Model Selection Involving Many Candidate ARMA Models. Computational Statistics and Data Analysis, 51, 6180-6196. http://dx.doi.org/10.1016/j.csda.2006.12.044

[3] Li, Z.Y. and Li, D.G. (2008) Strong Approximation for Moving Average Processes under Dependence Assumptions. Acta Mathematica Scientia, 28, 217-224. http://dx.doi.org/10.1016/S0252-9602(08)60023-5

[4] Box, G.E.P., Jenkins, G.M. and Reinsel, G.C. (1994) Time Series Analysis: Forecasting and Control. 3rd Edition, Prentice-Hall, Englewood Cliffs.

[5] Okereke, O.E., Iwueze, I.S. and Johnson, O. (2013) Extrema of Autocorrelation Coefficients for Moving Average Processes of Order Two. Far East Journal of Theoretical Statistics, 42, 137-150.

[6] Al-Marshadi, A.H. (2012) Improving the Order Selection of Moving Average Time Series Model. African Journal of Mathematics and Computer Science Research, 5, 102-106.

[7] Adewumi, M. (2014) Solution Techniques for Cubic Expressions and Root Finding. Courseware Module, Pennsylvania State University, Pennsylvania.

[8] Okereke, O.E., Iwueze, I.S. and Johnson, O. (2014) Some Contributions to the Solution of Cubic Equations. British Journal of Mathematics and Computer Science, 4, 2929-2941. http://dx.doi.org/10.9734/BJMCS/2014/10934

[9] Wei, W.W.S. (2006) Time Series Analysis, Univariate and Multivariate Methods. 2nd Edition, Pearson Addision Wesley, New York.

[10] Chatfield, C. (1995) The Analysis of Time Series. 5th Edition, Chapman and Hall, London.

[11] Palma, W. and Zevallos, M. (2004) Analysis of the Correlation Structure of Square of Time Series. Journal of Time Series Analysis, 25, 529-550. http://dx.doi.org/10.1111/j.1467-9892.2004.01797.x

[12] Iwueze, I.S. and Ohakwe, J. (2011) Covariance Analysis of the Squares of the Purely Diagonal Bilinear Time Series Models. Brazilian Journal of Probability and Statistics, 25, 90-98. http://dx.doi.org/10.1214/09-BJPS111

[13] Iwueze, I.S. and Ohakwe, J. (2009) Penalties for Misclassification of First Order Bilinear and Linear Moving Average Time Series Processes. http://interstatjournals.net/Year/2009/articles/0906003.pdf

[14] Okereke, O.E. and Iwueze, I.S. (2013) Region of Comparison for Second Order Moving Average and Pure Diagonal Bilinear Processes. International Journal of Applied Mathematics and Statistical Sciences, 2, 17-26.

[15] Montgomery, D.C., Jennings, C.L. and Kaluchi, M. (2008) Introduction to Time Series Analysis and Forecasting. John Wiley and Sons, New Jersey.

[16] Sittinger, B.D. (2010) The Second Derivative Test. www.faculty.csuci.edu/brian.sittinger/2nd_Derivtest.pdf 
Scientific Research Publishing (SCIRP) is one of the largest Open Access journal publishers. It is currently publishing more than 200 open access, online, peer-reviewed journals covering a wide range of academic disciplines. SCIRP serves the worldwide academic communities and contributes to the progress and application of science with its publication.

Other selected journals from SCIRP are listed as below. Submit your manuscript to us via either submit@scirp.org or Online Submission Portal.
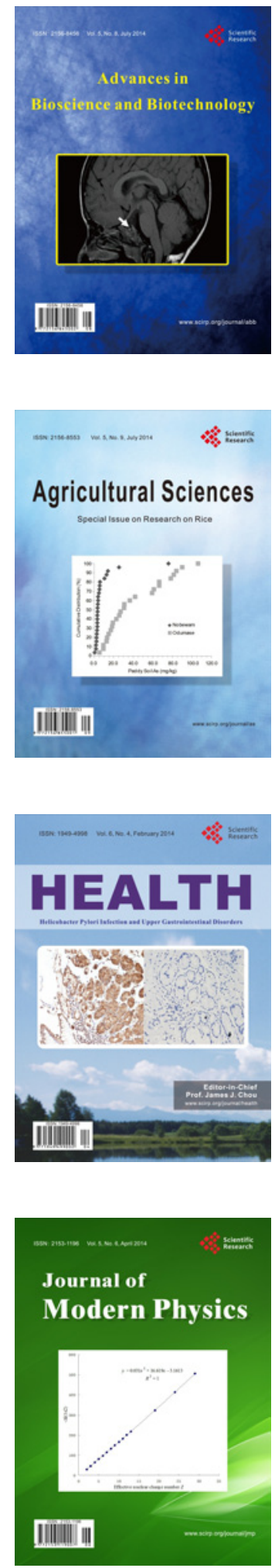
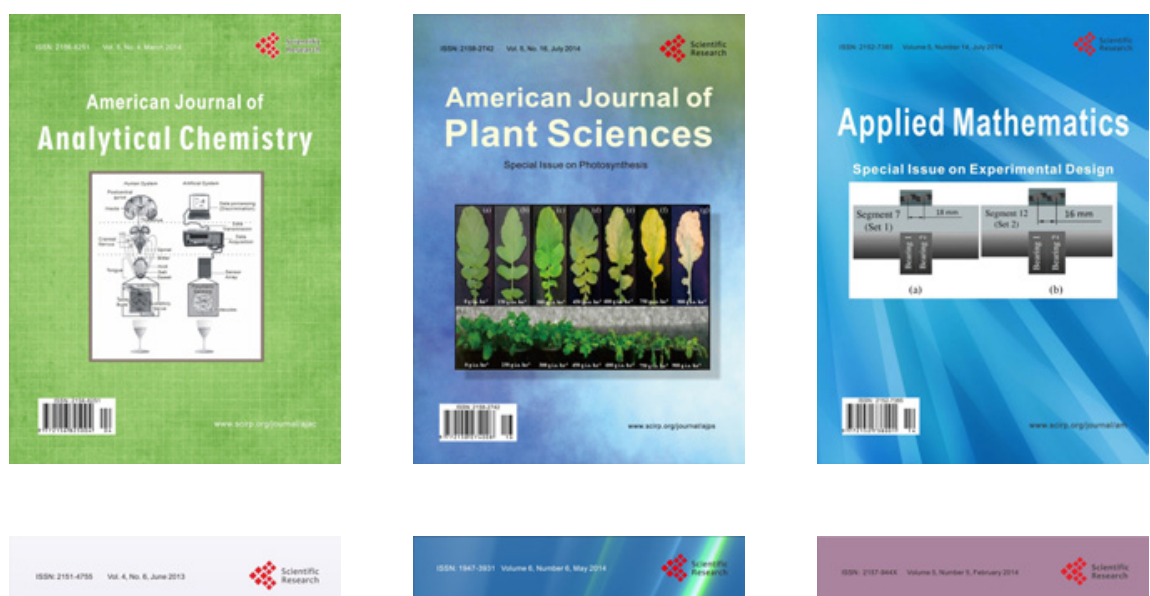

Creative Education
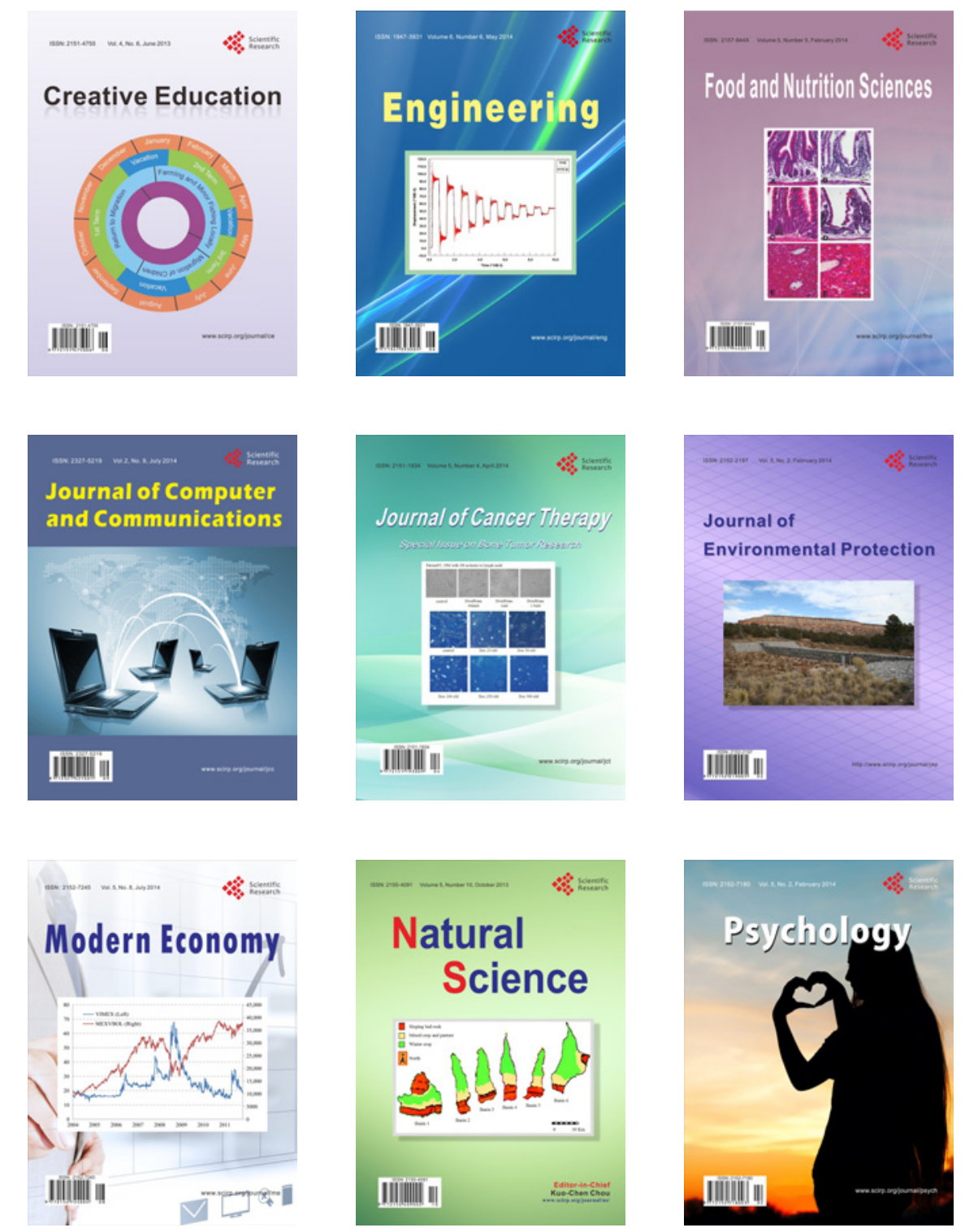